psychopraxis.neuropraxis 2014 - 17:1 DOI 10.1007/s00739-014-0222-1

Online publiziert: 4. Dezember 2014 ๑) Springer-Verlag Wien 2014
Peter Fischer

Psychiatrische Abteilung, Donauspital Wien, Wien, Österreich

\section{Beziehungsarbeit in der Psychiatrie}

re Aufenthaltsdauer beträgt österreichweit unter drei Wochen. Eigene Berechnungen der notwendigen Ärztezahlen an der Psychiatrie im Donauspital, errechnet aus jährlichen Patientenzahlen in den verschiedenen Diagnosegruppen und unter der Annahme, dass die Arbeitszeit der Ärzte zu $80 \%$ in der direkten Patientenbetreuung verbracht wird (restliche $20 \%$ für Fortbildung, Organisation, Diensteinteilung, Dienstübergaben, UbG-Verhandlungen etc.) zeigen einen Bedarf von genau 42 Ärzten pro 100 versorgungspsychiatrischen Betten, also exakt den Ärzteschlüssel anderer medizinischer Fächer.

Der Mangel an Fachärzten für Psychiatrie in Österreich, im meiner Meinung nach interessantesten Fach der gesamten Medizin, resultiert also nicht nur aus schlechter Bezahlung und praktisch fehlenden Sonderklassegebühren, sondern auch aus den Arbeitsbedingungen, die für die einzelnen Fachärzte in der Versorgungspsychiatrie frustrierend sind, da es keine Zeit für Beziehungsarbeit in Gesprächen mit Patienten und Angehörigen gibt.

\section{) Die Arbeitsbedingungen sind für Fachärzte in der Versorgungspsychiatrie frustrierend, da es keine Zeit für Beziehungsarbeit gibt.}

Vor diesem Hintergrund droht nun dank neuem Arbeitszeitgesetz mit besseren Arbeitsbedingungen auch für psychiatrisch tätige Ärzte, dass noch weniger Zeit für die Kommunikation mit unseren schwer kranken Patienten bleibt. Die Zeit für Beziehungsarbeit wird sich ab Jänner 2015 weiter verringern und die Patienten müssen mit noch stärker wechselnden
Ärzten vorlieb nehmen. Die mangelnde ärztliche Ausstattung der Versorgungspsychiatrie aus Zeiten der Stellenvermehrung in anderen Fachbereichen wird sich in Zeiten des Sparens und Bankenrettens die Errungenschaften des Fachs Psychiatrie und medizinische Psychotherapie mehr als wettzumachen.

Ihr

Peter Fischer

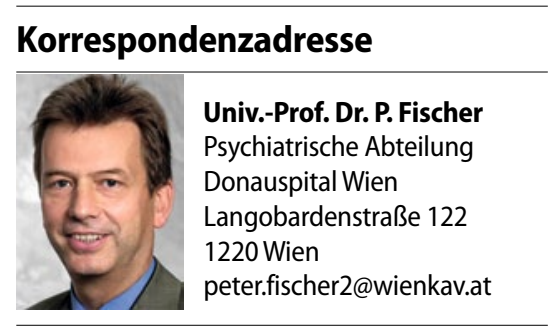
nicht mehr ausgleichen lassen. Dies droht
Dabei hat die Psychiatrie trotz schlechter Personalausstattung im ärztlichen Bereich seit Ende des 2. Weltkriegs enorme therapeutische Fortschritte gemacht. Auch die psychiatrischen Spitäler haben sich, von der Öffentlichkeit unbemerkt, zu Akutspitälern gewandelt. Die mittle- 